\title{
Vorwort zur zweiten Auflage
}

Bereits wenige Monate nach dem Erscheinen der ersten Auflage der „DROGENKUNDE“ mußten die Vorarbeiten für eine zweite Auflage in Angriff genommen werden. Die zustimmenden Urteile aus allen in Frage kommenden Kreisen haben den Grundgedanken, der das Buch schuf, in vollem $\mathrm{Maße}$ gerechtfertigt. Nicht nur die deutschen Fachkreise, sondern auch das Ausland bekundete, daß durch die „DROGENKUNDE“ eine Lücke in der Fachliteratur geschlossen wird. Ich danke an dieser Stelle allen Fachleuten für die freundliche Aufnahme meiner Arbeit und die mir übermittelten Anregungen, um die ich auch in Zukunft bitte. Eine besondere Freude war es mir, auch bei dem Nachwuchs unseres Berufsstandes ein großes Interesse festzustellen. Es wird auch in Zukunft mein Bestreben bleiben, die Ausbildungsarbeit an der Jugend mit allen Kräften zu unterstützen.

Neue Erkenntnisse auf unserem Fachgebiet konnten bei der Neuauflage verwertet werden. Neben anderen Arbeiten wurde die 6. Ausgabe des Ergänzungsbuches zum Deutschen Arzneibuch von I94I berücksichtigt. Die Angaben über die Anwendung wurden der maßgeblichen Literatur entnommen.

Da sich Herr Universitätsprofesșor a. D. Dr. Willy Peyer, J.eobschütz, liebenswürdigerweise bereit erklärte, an der Herausgabe der zweiten Auflage mitzuarbeiten, konnten wertvolle Bereicherungen aufgenommen werden. In dankenswerter Weise stellte mir Hẹrr Professor Peyer die Ergebnisse seiner eigenen Forschungen und seiner großen Erfahrung zur Verfügung. - Auch für die schwierige Arbeit des Korrekturlesens schulde ich ihm aufrichtigen Dank.

Ich danke weiterhin Herrn Erich Blembel i. Fa. Blembel Gebrüder, Hamburg, für die hervorragende Unterstützung, die er meiner Arbeit stets angedeihen lie $B$.

Trotz der eingeschränkten Möglichkèiten ist es auch jetzt wieder dem Verlag gelungen, für eine gute Ausstattung des Buches Sorge zu tragen. Für das größte Entgegenkommen allen meinen Wünschen gegenüber möchte ich meinen Verlegern an dieser Stelle meinen Dank abstatten.

Ich hoffe, da $B$ die neue Auflage ebenfalls den Beifall der Fachkreise finden und dazu beitragen wird, die schwierige Arbeit der Praxis zu erleichtern.

Hamburg, im August r942

\section{Heinz A. Hoppe}

\section{Vorwort zur dritten und vierten Auflage}

Die letzten Auflagen der ,DROGENKUNDE“ wurden wiederum sehr rasch verkauft $\mathrm{Da}$ der Verfasser zur Wehrmacht einberufen ist, haben wir die dritte und vierte Auflage mit Ausnahme kleiner Berichtigungen unverändert abgedruckt.

Ha mburg, im Juni r943/44 